Journal of Engineering and Applied Sciences 14 (7): 2222-2229, 2019

ISSN: 1816-949X

(C) Medwell Journals, 2019

\title{
Smart Robot Controlled via. Speech and Smart Phone
}

\author{
Sura F. Ismail, Ali W. Essa and Ahmed M. Ahmed \\ Department of Informatics Systems Management, \\ University of Information Technology and Communications, Baghdad, Iraq
}

\begin{abstract}
Robots are most valuable when they can effectively replace human involvement for many different things. The advancement of robotics and it's benefits in productivity, safety and in saving time and money caused them to become more widespread across various trends such as manufacturing and health care. Current direction is to build a walking robot that help elderly people. Therefore, in this study a smart humanoid voice controlled robot is implemented. A humanoid robot will serve the humanity, especially, the elderly people with at most efficient and reliable way. This robot can effectively replace human involvement for many different things. The robot in this study is implemented using 3D printing technology and controlled by smart phone using Bluetooth technology. The voice from the elderly person is process though proposed phone application to text then a command has been send to the brain of robot which is Arduino microcontroller to gives the correct action to the robot. Also the proposed robot monitors the patients using pulse sensor to keep them safe and also, avoid the obstacles using radar sensor. So, this robot can monitoring, doing tasks and make different conversations.
\end{abstract}

Key words: Robotics, voice control, smart phone, elderly people, Arduino, Bluetooth

\section{INTRODUCTION}

Creating machines that behave like humans and recognize the human speech and responding accordingly has been a human dream. With the improvements of the artificial intelligent and speech recognition technologies this dream comes true relatively. The robot system contains sensors, manipulators, control systems, power supplies working under programming software to perform a task. Therefore building a robot means engaging with Electrical, Mechanical and Structural Engineering, Mathematics, Physics and Computing. In some cases Biology, Medicine, Chemistry might also, be involved (Ramdhave and Joshi, 2016).

As robots become more cost effective at their jobs and as human labor continue to become more expensive, more and more industrial jobs become candidates for robotic automation. This is the single most important trend propelling growth of the industrial robot market. A secondary trend is that, economics aside as robots become more capable they become able to do more and more tasks that might be dangerous or impossible for human workers to perform.

The goal of the service robot is to help elder people in everyday life at social context through Speech Recognition (SR) algorithm which is an easy way of communication with human and it also, gives the advantage of interacting with the novice users without a proper training (Ozdemircan, 2008). A humanoid service robot which is a human-like shape robot is the default and preferred robot for most people. It must be noticed that it is difficult to claim that robots should be humanoid robots which are supposed to do some tasks in the real world, considering that aircrafts do not look like birds. The required functions for a robot may determine the optimal shape of the robot (Khaewratana, 2016).

By the technology and the humanoid robot functions this would help efficiency a great deal by reducing the needs of a human to serve the elderly people, since, the robots never get tired, never sleep and never eat, so, robots will save the time and money. Robotics in healthcare is so, much more than just movable metallic machines. With a remote controlled robot (voice commands) there will be an interaction between the elderly and the robot by using the smart watch or smart phone in the hand of the elderly people, the elderly will give the orders to the robot, the robot will implement the orders to serve them. Therefore, in this study a smart humanoid robot with all required components and technologies is proposed.

Litreature review: Number of studies and researches have been accomplished related to robot controlling

Corresponding Author: Sura F. Ismail, Department of Informatics Systems Management, University of Information Technology and Communications, Baghdad, Iraq 
through speech recognition and smart devices. The following presents some of these researches Yokoyama et al. (2003) developed a humanoid robot named HRP-2P that demonstrated a capability of working hand-to-hand with human operators in a construction work. While the robotic arm developed by Holada and Pelc (2008) is based on a former research. This research involved a voice-control dialog system, speech recognition and vocabulary design and speech synthesis feedback for user command confirmation. Together with a scene manager and a digital image processing module, it forms the core of the control system.

Winter (2013) has developed a low cost and open source philosophy android controlled mobile robot. Holada and Pelc (2008) has proposed a robotic system attached with wireless camera for the purpose of surveillance via. Bluetooth technology. Guardi (2014) has proposed a design of a Bluetooth technology. Android application for microcontroller driven robot. The main objective of his research is to show that a single android application is capable of working with different electronic devices typically used within the hobby and armature robotic field.

Selvam (2014) have developed android controlled robotic architecture for pick and retaining of objects. Main objective of their research was creating a pick and retain robot which can be used for bomb diffusion from safe distance. Also, the researchers used the smart watch technology to control robotics remotely using some smart watch applications, through the Bluetooth or Wi-Fi technology (by making a network that includes the parts or robotics to be controlled) (Lutze and Waldhor, 2016).

\section{MATERIALS AND METHODS}

Proposed system: The goal of this project is to build a smart humanoid robot that can be controlled remotely using voice commands. Ash robot application is proposed here, to control the robot which is called "Ash Robot". In this application a speech will convert into text then send to the robot which interprets it and make the correct action (for example go forward). The speech recognition software using in this application can analyze the sounds by filtering and digitizing it to a format to be read and analyzing its meaning. Then, based on algorithms and previous input it can make a highly accurate educated guess.
The problem of a background noise that throw the speech recognition device off track is appears because it does not inherently have the ability to distinguish the ambient sound, so, the solution to this problem is to use a voice recognition algorithm designing to reduce the noise to $70 \%$ and also, a microphone has been used for better quality and response time. A general model for all the processes occur in the proposed project is shown in Fig. 1.

Hardware design: The robot's hardware design has a huge influence in order to obtain a reliable biped robot. The mechanical structure design must always attempt an adequate robot's proportions. For that reason, mass distributions, CM (Center of Mass) location and the actuators selection are important stages on the mechanical structure design and have a direct impact on the robot's performance. Also, here a modular with extra design for an easy robot's configuration links is proposed to allow quick changes on the structure. And the electronic design must mainly be fast enough to perform the control algorithms, handle some sensors and intercommunication with human using mobile devices. Finally and in general, the robot's hardware design must keep on mind add less weight as possible and been low cost.

Ash robot: Humanoid robot must design in a way that a body shape looks like the human body, so, the robot body here is making by using 3D printing. The robot body includes head, manikin human body, two legs moving through wheels and two arms connected to the body. Head has been printed using 3D printer it contains two servos motor and speaker. One of the servos is to move the jaw and the other for moving neck right and left in $90^{\circ}$ rotation and reverse it. It also contains the Light-Emitting Diode (LED) for eyes. Like human head the printing parts of head contain mouth, eyes, jaw, neck etc. as shown in Fig. 2.

The moving wheel is the most common moving element which provides the robot with a suitable speed, accuracy and stability. It look like hover board that has 4 wheels, the first two wheels at right and left for moving and it is 9 inch wheels, the other two wheels are 5 inch wheels at the front and back. The reason for using 4 wheels is to make it stable and avoid obstacles. Each larger wheel connected to $18 \mathrm{~V} \mathrm{DC}$ drill motor that has high torque, very fast and can handle large weight. The DC motors connected to high current motor driver (IBT2) is actually an H-bridge driver based on the Infineon chips 


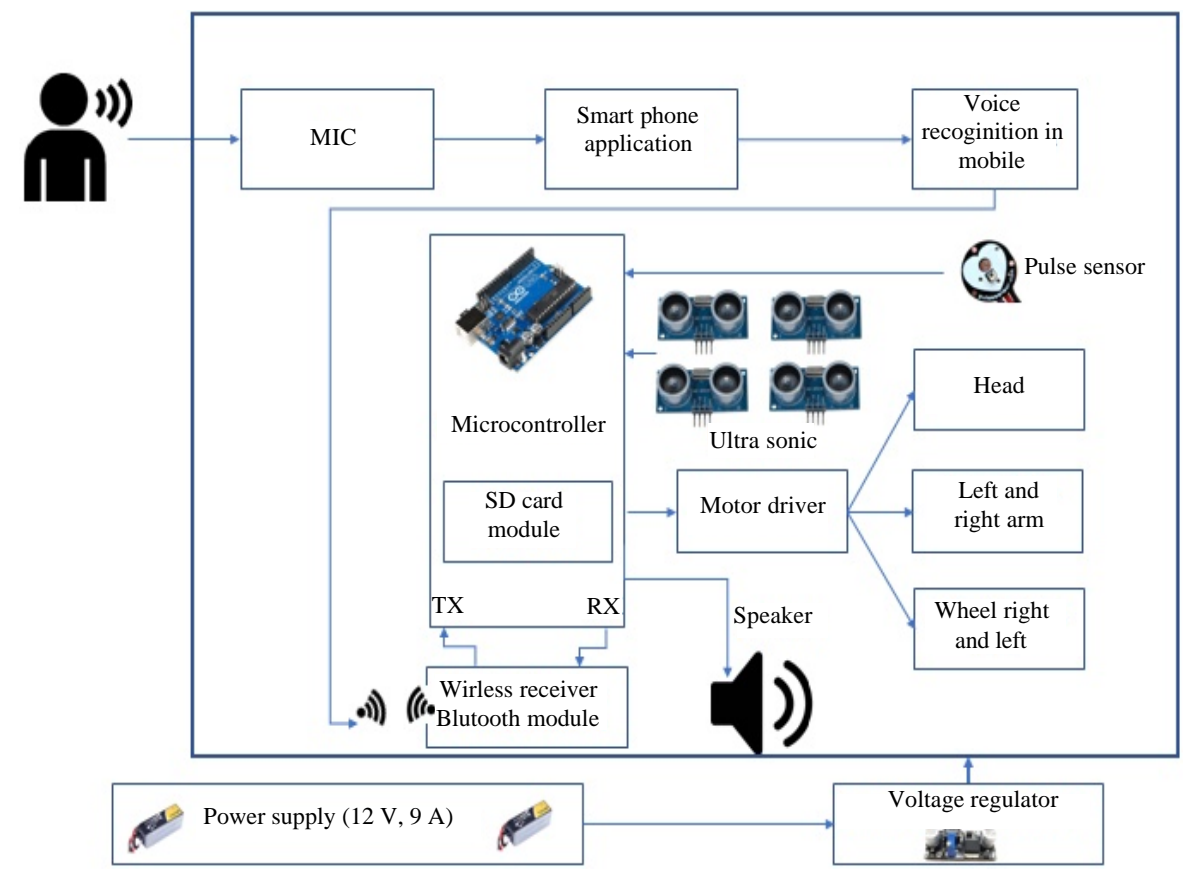

Fig. 1: General model for the proposed robot (Ash robot)

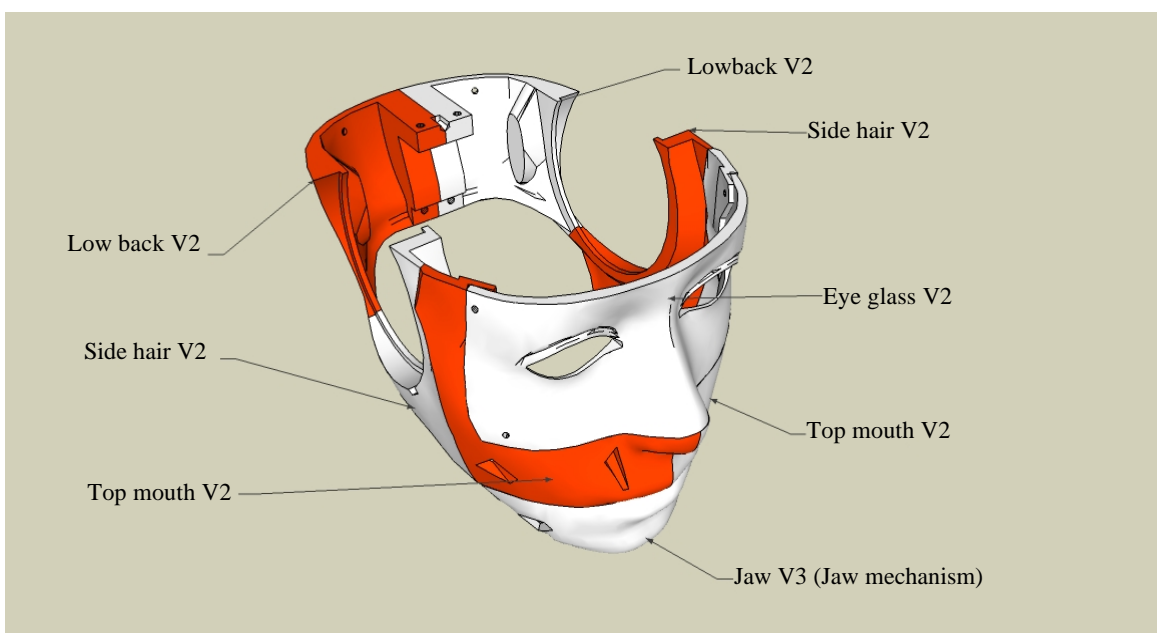

Fig. 2: Head of Ash robot

BTS7960. While the robot arm is a type of mechanical arm programming with a similar human arm functions. The two arms also created using 3D printing and it has three servos motors, the first for base rotation, the second for shoulder rotation and the third one is for catching things and it is a gripper.

Microcontroller: Controlling ash robot and building the brain needs a programmable electronic chip an Arduino Mega is the best choice due to its suitable storage area, digital and analog input/output pins. Also, Arduino in general is an open-source electronics platform based on easy-to-use hardware and software.

Sensors: A sensor is a device that detects and responds to some type of input from the physical environment. In this study, two types of sensors had been used to achieve the Ash robot specification; Ultrasonic sensor and heart pulse sensor. 


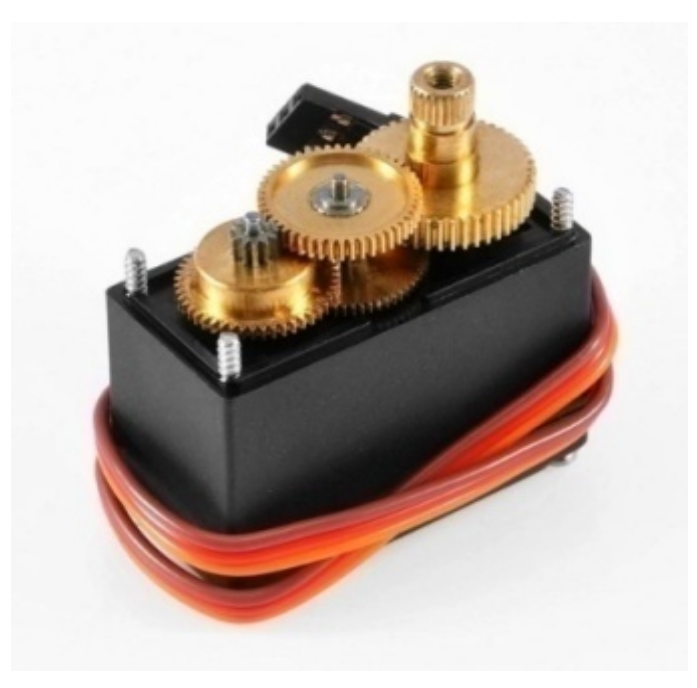

Fig. 3: MG995 servo motor

Servos: Servo motors have been around for a long time and are utilized in many applications. It is used to operate remote-controlled or radio-controlled toy cars, robots and airplanes. The servos type that had been used in this study is MG995 Metal Gear servo and rotates to $180^{\circ}$ at operation voltage: $4.8-7.2 \mathrm{~V}$ as shown in Fig. 3.

SD card module: A breakout board used for SD card processes such as reading and writing with a microcontroller and it is compatible with microcontroller systems like arduino. In this study, this module is used to read voice data from micro RAM that store all related wav. format voice of robot.

Bluetooth HC-05: HC-05 module is an easy to use Bluetooth SPP (Serial Port Protocol) module designed for transparent wireless serial connection setup. The HC-05 Bluetooth module can be used in a master or slave configuration, making it a great solution for wireless communication. In the case of slave, the modules cannot initiate a connection to another Bluetooth device but can accept connections while master module can initiate a connection to other devices. In this project it acts as slave, since, smart phone will connect to robot and commands it.

Microphone and speaker: A microphone is a device that captures audio by converting sound waves into an electrical signal. While the speaker is a device that outputs the sound saved in the SD card. The sound command will sent to the speaker from the output pins of

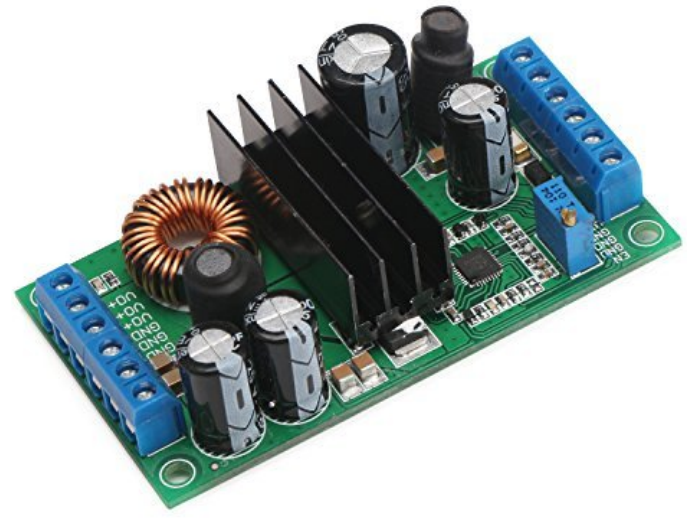

Fig. 4: A step down voltage regulator

the Arduino Mega. It through AUX cable. In other words, microphone for input (from patient to robot) while speaker for output (from robot to patient).

Voltage regulator: A voltage regulator is the preferred device used to provide the robot with a steady, reliable voltage. There are different types of voltage regulator but in this study a step down switching voltage regulator has been used which converting from $12-5 \mathrm{~V}$ as shown in Fig. 4. This voltage regulator is used for servos, sensors and Bluetooth chips.

Power supply: All components that listed above need a power supply, so, in this study two batteries with $12 \mathrm{~V}$ and current of 9 ampere are used. The first one is for DC drill motors and the other for all necessary components.

Software design: Robot software design is the set of programming commands or instructions that tell a robot about what tasks to perform. Many software systems and frameworks have been proposed to make programming the robot more easier task. In this study the Arduino Microcontroller is programming through Arduino Software which is open-source software and the smart phone that used to control robot though Bluetooth is programming through a simple powerful application using MIT (Massachusetts Institute of Technology) App inventor. App. Inventor lets developer to develop applications for Android phones using a web browser and either a connected phone or emulator. The proposed project; Ash robot application used two things; the Bluetooth connection and the voice recognition that provided by Google. Finally, a general flowchart that described the basic processes in the Ash robot will illustrated in Fig. 5. 


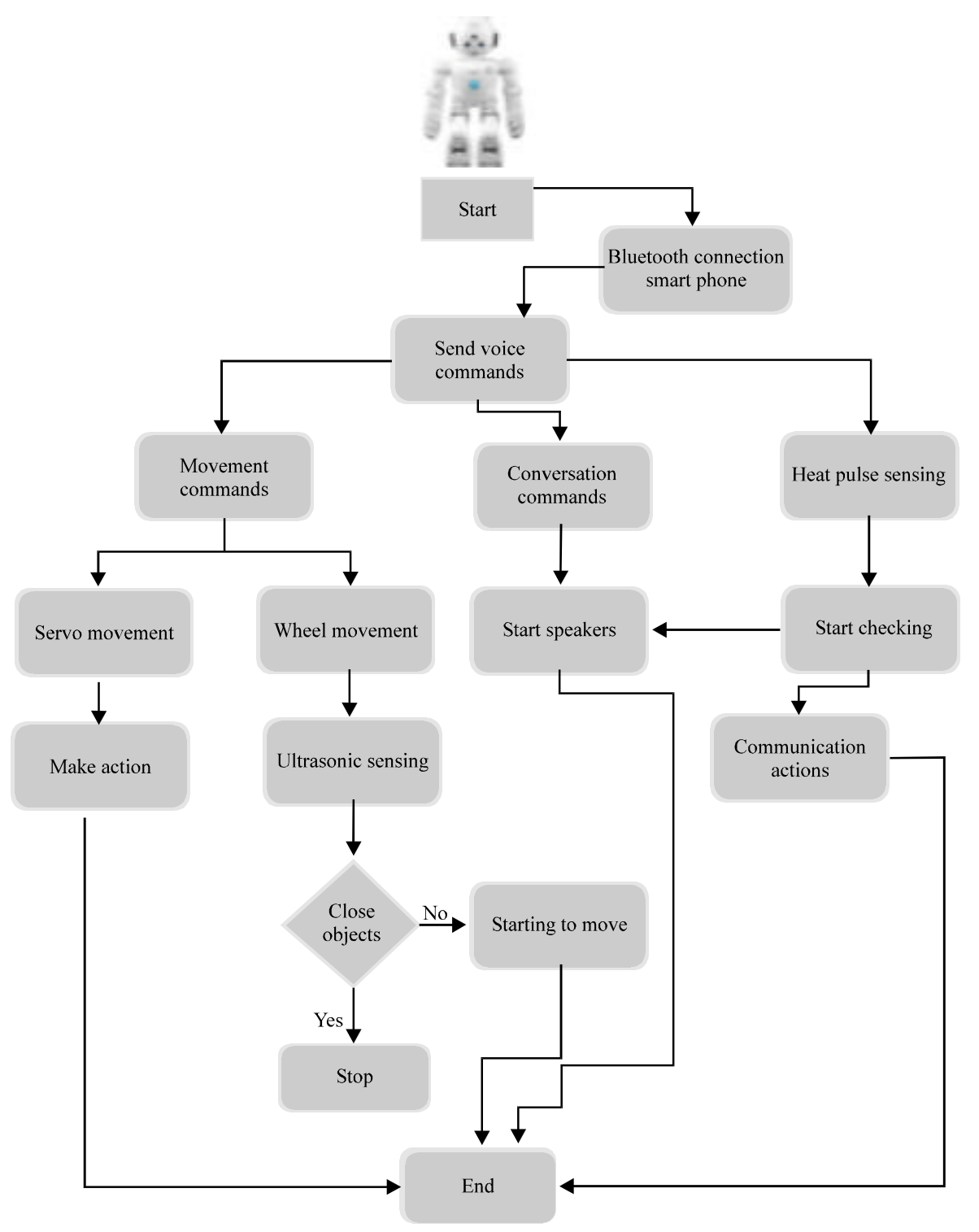

Fig. 5: A general flowchart for the proposed robot (Ash robot)

\section{RESULTS AND DISCUSSION}

As mentioned before, the Ash robot has a humanoid structure like hands, head, body and legs, standing on hover board for movement. Some of the materials made by using 3D printing technology (head and hands), the body made of Manikin and the hover board made of iron board connected to 4 wheels (right and left connected to DC motors for the actual movement, the front and back wheels to balance the Ash robot).

Also, there are two sensors in this robot, one for making distance measurement to avoid obstacles (using ultrasonic sensor) and heart pulse sensor for measuring the heart pulse putting on legs on the body and eyes to have a better looking. Servo also, used for making the hands and head movements; two servos for head and four servos for two hands. Illustration pictures of Ash robot are show in Fig. 6 and 7.

Ash robot tasks: The Ash robot is able to do the following tasks or operations.

Controlling: The Ash robot is controlling through voice commands that received and processed by the smart phone Ash robot application that created and programmed specifically for this robot. When turning on 

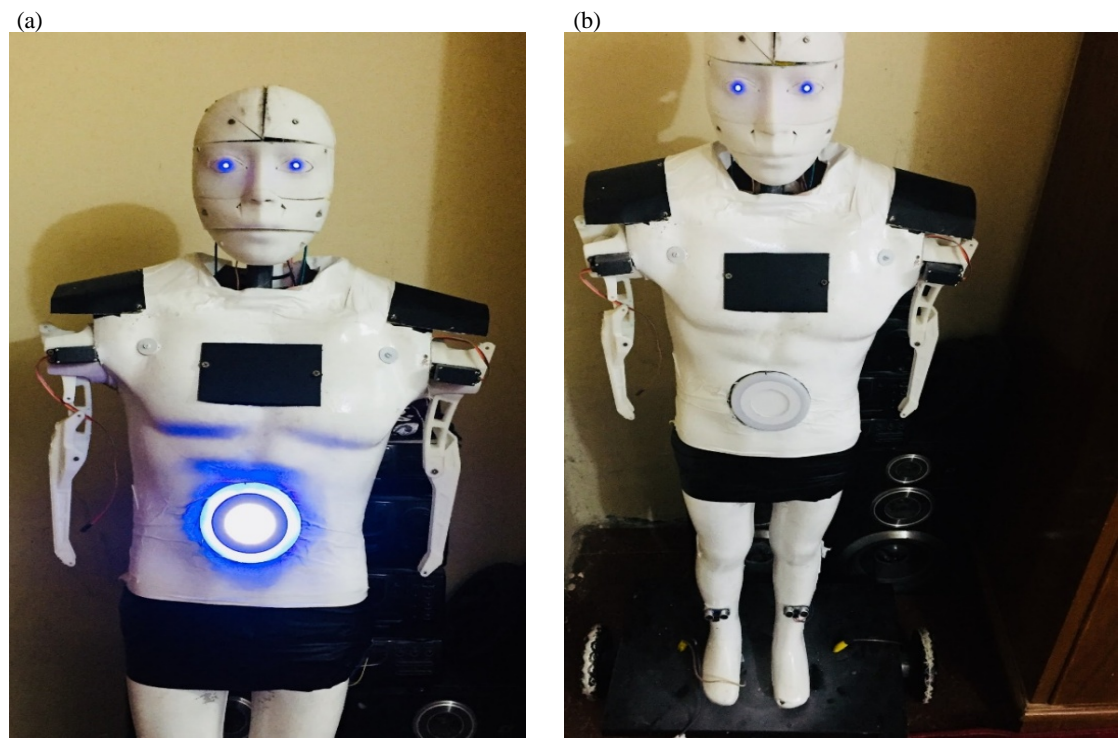

Fig. 6: a, b) Ash robot

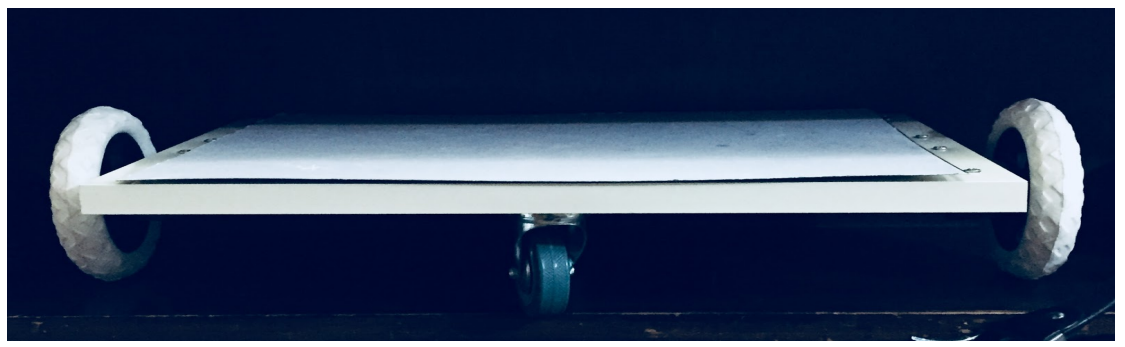

Fig. 7: Hover board for Ash robot standing

(a)

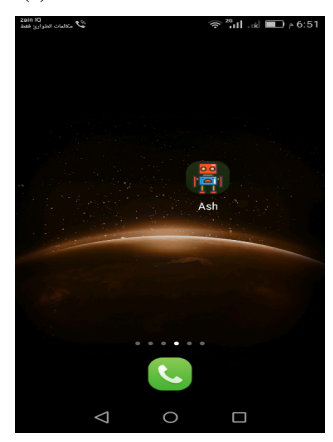

(b)

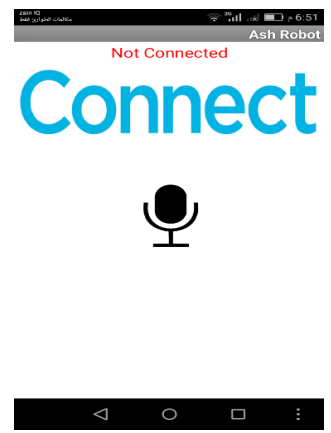

(c)

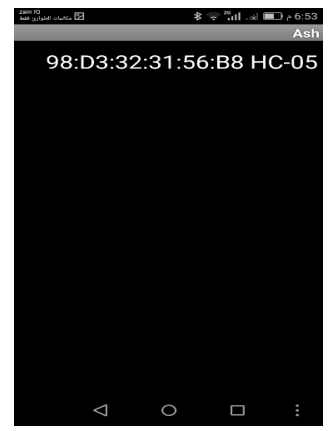

Fig. 8: a-c) Ash robot application for android (connection)

the Ash robot, the Bluetooth $\mathrm{HC}-05$ module will turn on in the robot if this module blinks continuously in a periodic manner, that means there is no connection available while when it gets connected to any Bluetooth device, its blinking slows down to $2 \mathrm{sec}$. Therefore to connect the Ash robot, open the application and the name of module will show on the list with the label on the main interface change to "is connected". After the connection has been made then the voice icon must be tapped to receive the voice and convert the voice to text. The recognized text from the voice will show on the label of the application and automatically this text is send to the Ash robot to do the required job as shown in Fig. 8 and 9. 
Monitoring: Another task doing by robot is monitoring the heart rate of the elder person through a plug-and-play heart-rate sensor which is pulse sensor amped as shown in Fig. 10. The heart rate data is read when person place his finger on the pulse lightly for a little of time then the data processed and a notification voice message will send from Ash robot to person for example "your heart pulse is stable there is no danger". Another monitoring sensor is ultrasonic sensor which is used to measure the distance and change the direction of Ash robot or stop it if there are any objects around $100 \mathrm{~m}$ as shown in Fig. 11.

Conversations: Ash robot can make a conversation with the elder person and depend on the commands sending from person to robot a correct action is occurring.
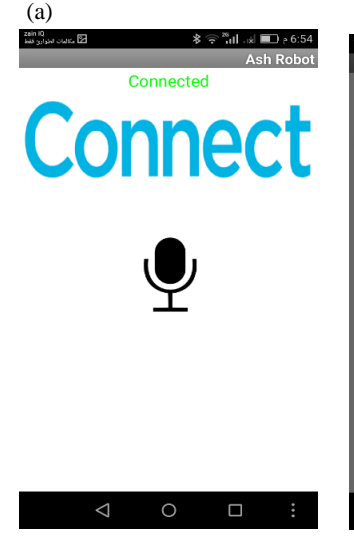

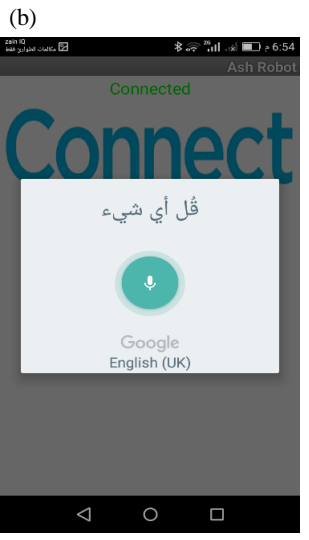

(c)

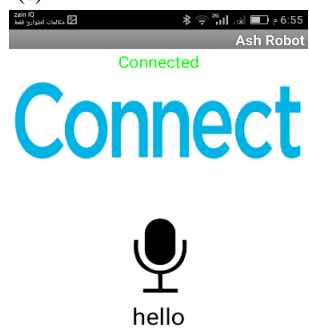

$\triangleleft \quad \circ \quad \square$

Fig. 9: a-c) Ash robot application for android (sending voice commands)

(a)

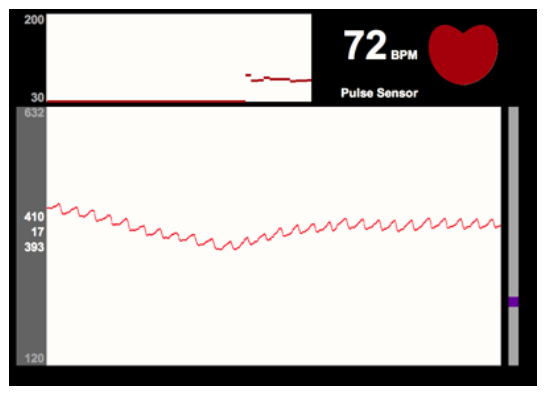

(b)

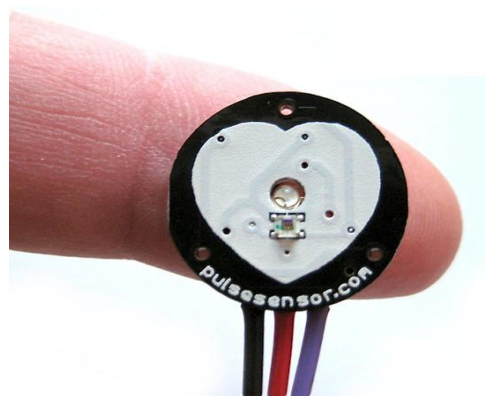

Fig. 10: a, b) Pulse sensor monitoring test

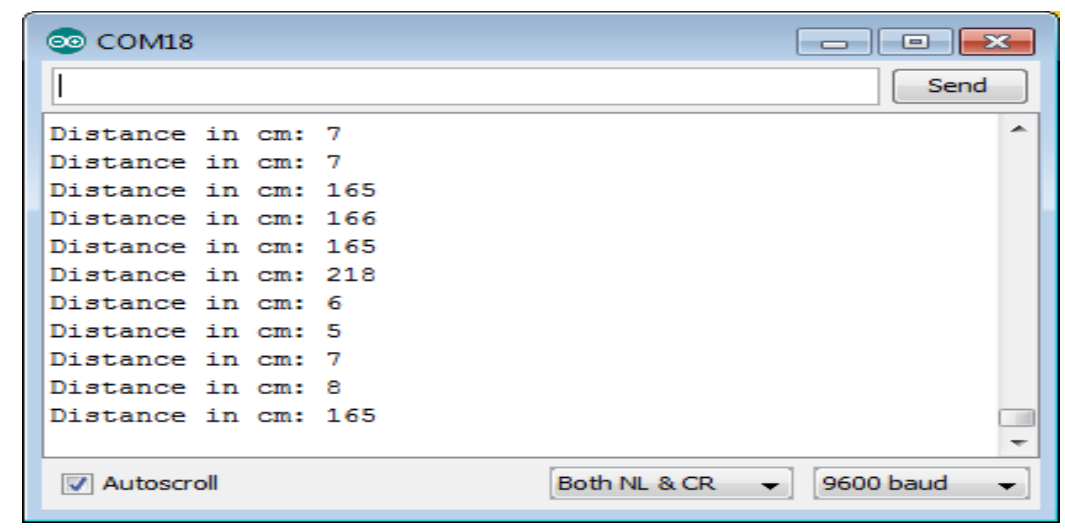

Fig. 11: Ultrasonic distance test 


\section{CONCLUSION}

Ash robot is a humanoid smart voice controlled robot that replace the human effort to make tasks and help elder people by using a common technologies for connection, programming the robot using a common programming language $\mathrm{c}$ with a well known microcontroller Arduino and by using the technique of transform voice into readable text data to make several actions such as making movement on several component like the head can turn right and left, the hands can move up to down using servos and also front to back and wheels that robot stands on. Also, to avoid the human mistakes, the Ash robot has a radar sensor (Ultrasonic sensor) to make self processing if there are any objects to avoid obstacles and also, making alert if there is something nearly. Also, this robot helps elder person by measuring their heartbeat and telling them about the heartbeat results and if they are in safe mode or not. Finally, robots cannot perform every job today robots roles include assisting research and industry and as the technology improves there will be new ways to use robots which will bring new hopes and new potentials.

\section{REFERENCES}

Guardi, V.M., 2014. Design of a Bluetooth enabled android application for a microcontroller driven robot. $\mathrm{Ph} . \mathrm{D}$ Thesis, Rensselaer Polytechnic Institute, Troy, New York, USA.

Holada, M. and M. Pelc, 2008. The Robot Voice-Control System with Interactive Learning. In: New Developments in Robotics Automation and Control, Lazinica, A. (Ed.). IntechOpen, Rijeka, Croatia, ISBN:978-953-7619-20-6, pp: 219.
Khaewratana, W., 2016. Development of a voicecontrolled human-robot interface. Master Thesis, Department of Manufacturing and Mechanical Engineering Technology, Rochester Institute of Technology, Rochester, New York, USA.

Lutze, R. and K. Waldhor, 2016. The application architecture of smartwatch apps-analysis, principles of design and organization. Proceedings of the International Conference on Lecture Notes in Informatics (LNI), September 26-30, 2016, Gesellschaft fur Informatik, Bonn, Germany, pp: $1-13$.

Ozdemircan, M.Z., 2008. Robot control with voice command. Master Thesis, Department of Computer Engineering, Yýldýz Technical University, Istanbul, Turkey.

Ramdhave, S. and S. Joshi, 2016. Voice reconition robot control using android device. Intl. J. Adv. Comput. Eng. Networking, 4: 29-31.

Selvam, M., 2014. Smart phone based robotic control for surveillance applications. Intl. J. Res. Eng. Technol., 3: 229-232.

Winter, J.K., 2013. Android controlled mobile robot. Master Thesis, University Carlos III de Madrid, Getafe, Spain.

Yokoyama, K., H. Handa, T. Isozumi, Y. Fukase and K. Kaneko et al., 2003. Cooperative works by a human and a humanoid robot. Proceedings of the 2003 IEEE International Conference on Robotics and Automation (Cat. No.03CH37422), September 14-19, 2003, IEEE, Taipei, Taiwan, pp: 2985-2991. 